\title{
Octonacci photonic crystals with negative refraction index materials
}

\author{
E.R. Brandão a, M.S. Vasconcelos ${ }^{\text {b, }}{ }^{\text {, }}$, D.H.A.L. Anselmo ${ }^{\text {a }}$ \\ a Departamento de Física Teórica e Experimental, Universidade Federal do Rio Grande do Norte, 59072-970, Natal, RN, Brazil \\ ${ }^{\mathrm{b}}$ Escola de Ciências e Tecnologia, Universidade Federal do Rio Grande do Norte, 59072-970, Natal, RN, Brazil
}

\section{A R T I C L E I N F O}

\section{Article history:}

Received 19 October 2016

Accepted 10 November 2016

Available online 23 November 2016

\section{Keywords:}

Photonic crystals

Metamaterials

Transmission

Multilayers

\begin{abstract}
A B S T R A C T
We investigate the optical transmission spectra for $s$-polarized (TE) and p-polarized (TM) waves in onedimensional photonic quasicrystals on a quasiperiodic multilayer structure made up by alternate layers of $\mathrm{SiO}_{2}$ and metamaterials, organized by following the Octonacci sequence. Maxwell's equations and the transfer-matrix technique are used to derive the transmission spectra for the propagation of normally and obliquely incident optical fields. We assume Drude-Lorentz-type dispersive response for the dielectric permittivity and magnetic permeability of the metamaterials. For normally incident waves, we observe that the spectra does not have self-similar behavior or mirror symmetry and it also features the absence of optical band gap. Also for normally incident waves, we show regions of full transmittance when the incident angle $\theta_{C}=0^{\circ}$ in a particular frequency range.
\end{abstract}

(C) 2016 Elsevier B.V. All rights reserved.

\section{Introduction}

Recently, the idea of complex materials in which both the permittivity and the permeability possess negative real values at certain frequencies has received considerable attention. In 1967, Veselago theoretically investigated plane-wave propagation in a material whose permittivity and permeability were assumed to be simultaneously negative [1]. His theoretical study showed that for a monochromatic uniform plane wave in such a medium the direction of the Poynting vector is antiparallel to the direction of the phase velocity, contrary to the case of plane-wave propagation in conventional simple media. In recent years, Smith, Schultz, and their group constructed such a composite medium for the microwave regime and demonstrated experimentally the presence of anomalous refraction in this medium [2,3]. For metamaterials with negative permittivity and permeability, several names and terminologies have been suggested, such as "left-handed" media [1-7]; media with negative refractive index (NRI) [1-4,6]; "backwardwave media" (BW media) [8]; and "double-negative" (DNG) metamaterials [9], to name a few. Many research groups all over the world are now studying various aspects and applications of these materials which have been proposed.

Also, since the discovery of quasicrystals by Shechtman et al. in 1984 [10], many efforts have been conducted to understand the

\footnotetext{
* Corresponding author.

E-mail address: mvasconcelos@ect.ufrn.br (M.S. Vasconcelos).
}

physical properties of these aperiodic materials, which possess a long range order without having a translational symmetry. Hence, quasicrystals are regarded to have a degree of order intermediate between crystals and disordered systems. Quasicrystalline systems have been extensively studied, not only with respect to their structure, which shows uncommon rotational symmetries [10-12], and their electronic states, which were found to show a Cantor-set spectrum in one dimension [13-16], but also phonons and magnetic properties of these materials have been investigated [17-21]. Although the term quasicrystal is more appropriate when applied to natural compounds or artificial alloys, in one dimension (1D) there is no difference between this and the quasiperiodic structures formed by the incommensurate arrangement of periodic unit cells. An appealing motivation for studying such structures is that they exhibit a highly fragmented energy spectrum displaying a selfsimilar pattern.

Further, the photonic properties of those quasiperiodic structures are of special interest because the complex symmetries in quasicrystals make them suitable for the application in several optical devices such as single-mode light-emitting diodes, polarization switching and microelectronic devices that are based on photons rather than on electrons, which potentially can be the electromagnetic analogue to semiconductors [22-26]. The theoretical study of photonic properties of one-dimensional systems is based on the transfer matrix method and the concept of aperiodic mathematical sequences, as the Fibonacci sequence, the ThueMorse sequence, and Cantor sequences [23,27-29]. Such onedimensional systems can be relatively easily produced in reality 
and a comparison of the theoretical and the experimental results shows a good agreement [30,31]. For the theoretical study of quasiperiodic systems one often applies the concept of aperiodic mathematical sequences/tilings. Especially, the photonic properties of one-dimensional systems have been extensively analyzed with this approach, where transfer matrix methods can be applied. There are examples based upon the Fibonacci, Thue-Morse and Cantor sequences [23,27,28,32-34], and also systems with negative refractive indices have been studied [35-38,40,41].

It is the aim of this work to study the propagation of light waves in multilayer photonic structures composed of $\mathrm{SiO}_{2} /$ metamaterial (labeled $A$ and $B$ on this work) layers stacked alternately following the Octonacci sequence, which describes the arrangement of spacing of the Ammann quasilattice (8-grid), namely, the octagonal Ammann-Becker tiling [42]. The quasiperiodic structure follows the Octonacci sequence, and the multilayer photonic structure can be grown by juxtaposing the two building blocks $A$ and $B$, where the $n$ th-stage of the superlattice $S_{N}$ is given iteratively by the rule $S_{N}=S_{N-1} S_{N-2} S_{N-1}$, for $N \geq 3$, with $S_{1}=A$ and $S_{2}=B$. The number of the building blocks increases according to the Pell number, $P_{N}=2 P_{N-1}+P_{N-2}$ (with $P_{1}=1$ e $P_{2}=1$ ). This structure can also be grown by following a recurrence rule, namely: $A \rightarrow B, B \rightarrow B A B$. Recently, we have studied the transmission spectra in onedimensional photonic quasicrystals, made up of $\mathrm{SiO}_{2}$ (A) and $\mathrm{TiO}_{2}$ (B) materials, organized following the Octonacci sequence [43]. In that work we report, for normally incident waves and for a same generation, that the transmission spectra for transverse electric (TE) and transverse magnetic (TM) waves presents a perfect scaling property where a self-similar behavior is obtained, as an evidence that these spectra are fractals. Also we show regions where the omnidirectional band gaps emerges for specific generations of Octonacci photonic structure, except for TM waves. On the other hand, M.-R. Wu et al. [44], have studied theoretically the photonic bandgap structure for a polaritonic photonic crystal containing lithium tantalate $\left(\mathrm{LiTaO}_{3}\right)$ in the NRI region. They have concluded that in NRI region we have a multi band gap structure and that the gap falling in the anomalous dispersion region can be treated as a zero-index gap which is further shown to be omnidirectional. However in both works, the authors do not explore the NRI region in order to study the transmission spectra. It is one of our aims to fill this gap, by studying the optical transmission in this region in order to search perfect, or almost perfect, transmission peaks.

Specifically, in this paper we want to investigate the behavior of the light when it pass through an Octonacci photonic layered system, considering the central wavelength $\lambda_{0}=700 \mathrm{~nm}$ [45]. We also intend to investigate the influence of the oblique incidence at the system, by searching for frequency regions where the band gaps are independent from polarization and the incident angle $\theta_{c}$.

The plan of this work is as follows. In Sec. 2, we present the method of calculation employed here, which is based on the transfer-matrix approach, together with a discussion of the transfer matrices for the quasiperiodic structure presented here. In Sec. 3 we present our results and discuss them. This is followed by a brief conclusion in Sec. 4.

\section{Theoretical model}

In the present work we make use of a theoretical model based on a transfer-matrix treatment (for a review see Refs. $[38,40]$ ). Consider a $s$-polarized (TE wave) light of frequency $\omega$, normally incident from a transparent medium $C$ at an arbitrary angle $\theta_{C}$ with respect to the normal direction of the layered system (see Fig. 1). The layered system is formed from an array of slabs of different materials $(A$ or $B)$. The reflectance and the transmittance coefficients are simply given by

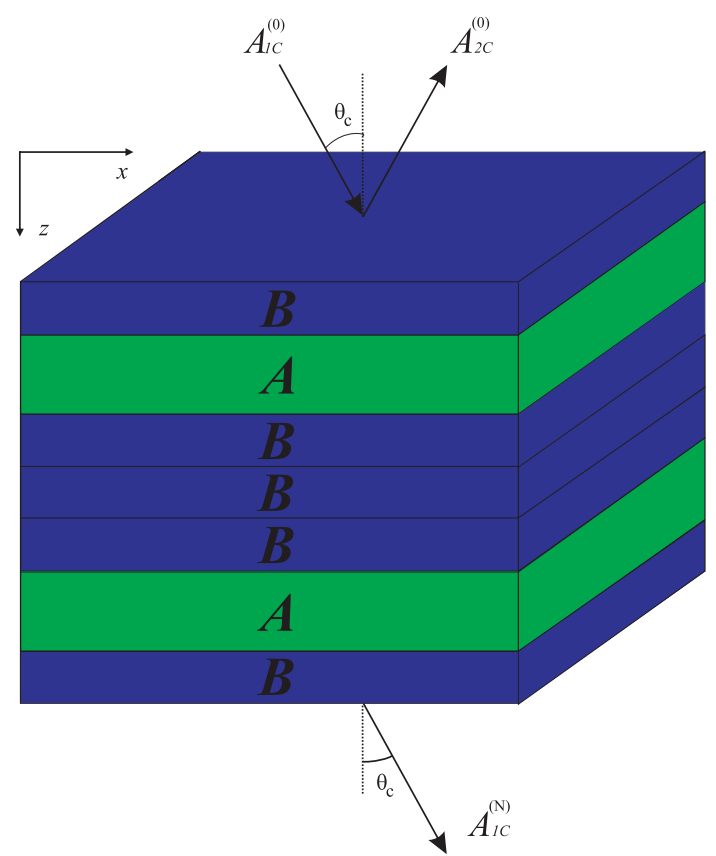

Fig. 1. Schematic representation showing the geometry of the Octonacci quasiperiodic multilayer system considered in this work. More precisely, for sequence $S_{4}=[B|A| B|B| B|A| B]$, with $P_{4}=7$. $L$ is the size of the whole superlattice structure.

$R=\left|\frac{M_{21}}{M_{11}}\right|^{2} \quad$ and $\quad T=\left|\frac{1}{M_{11}}\right|^{2}$,

where $M_{i j}(i, j=1,2)$ are the elements of the optical transfer-matrix $M_{N}$, which links the coefficients of the electromagnetic fields in the region $z<0$ to the coefficients of the electromagnetic fields in the region $z>L, L$ being the size of the quasi-periodic structure.

Let us consider first, to illustrate our method, the optical transfer-matrix calculation for the quasiperiodic multilayer which is characterized by having two dielectric media $A$ and $B$ with thicknesses $d_{A}$ and $d_{B}$, and refractive indexes $n_{A}$ and $n_{B}$, respectively, organized in accordance to the Octonacci sequence. It is surrounded by the transparent medium $C$ with refractive index $n_{C}$ (see Fig. 1). The transmission of an obliquely incident light wave across the interfaces $\alpha \rightarrow \beta$ (i.e., $C \rightarrow A, A \rightarrow B, \ldots, B \rightarrow C$ ) is represented by the matrix

$M_{\alpha \beta}=\frac{1}{2}\left[\begin{array}{ll}1+k_{z \alpha} / k_{z \beta} & 1-k_{z \alpha} / k_{z \beta} \\ 1-k_{z \alpha} / k_{z \beta} & 1+k_{z \alpha} / k_{z \beta}\end{array}\right]$

with

$k_{z \alpha}=\left[\left(n_{\alpha} \omega / c\right)^{2}-k_{x}^{2}\right]^{1 / 2}$

and

$k_{x}=n_{c}(\omega / c) \sin \left(\theta_{C}\right)$.

The propagation of the light wave within one of the layers $\gamma$ $(\gamma=A$ or $B)$ is characterized by the propagation matrices

$M_{\gamma}=\left[\begin{array}{cc}\exp \left(-i k_{\gamma} d_{\gamma}\right) & 0 \\ 0 & \exp \left(i k_{\gamma} d_{\gamma}\right)\end{array}\right]$

We assume that, in each layer, the electrical field is given by 


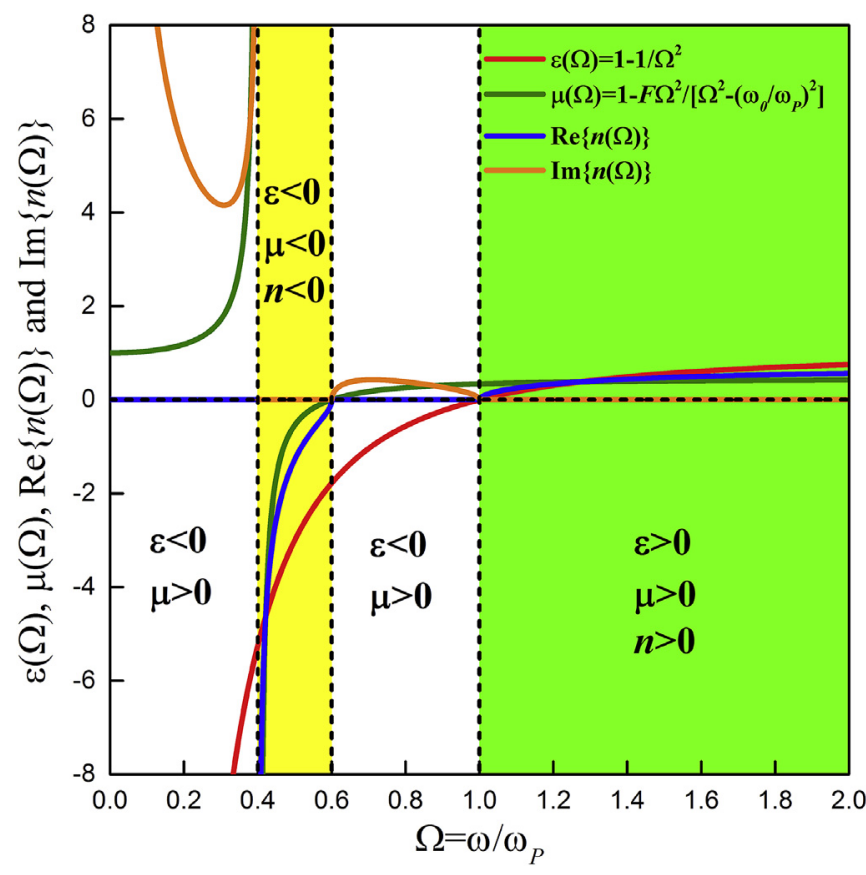

Fig. 2. Permittivity (red line), permeability (green line), real part of the refractive index (blue line) and imaginary part of the refractive index (orange line) of layer $A$ versus the reduced frequency $\Omega$. (For interpretation of the references to colour in this figure legend, the reader is referred to the web version of this article.)
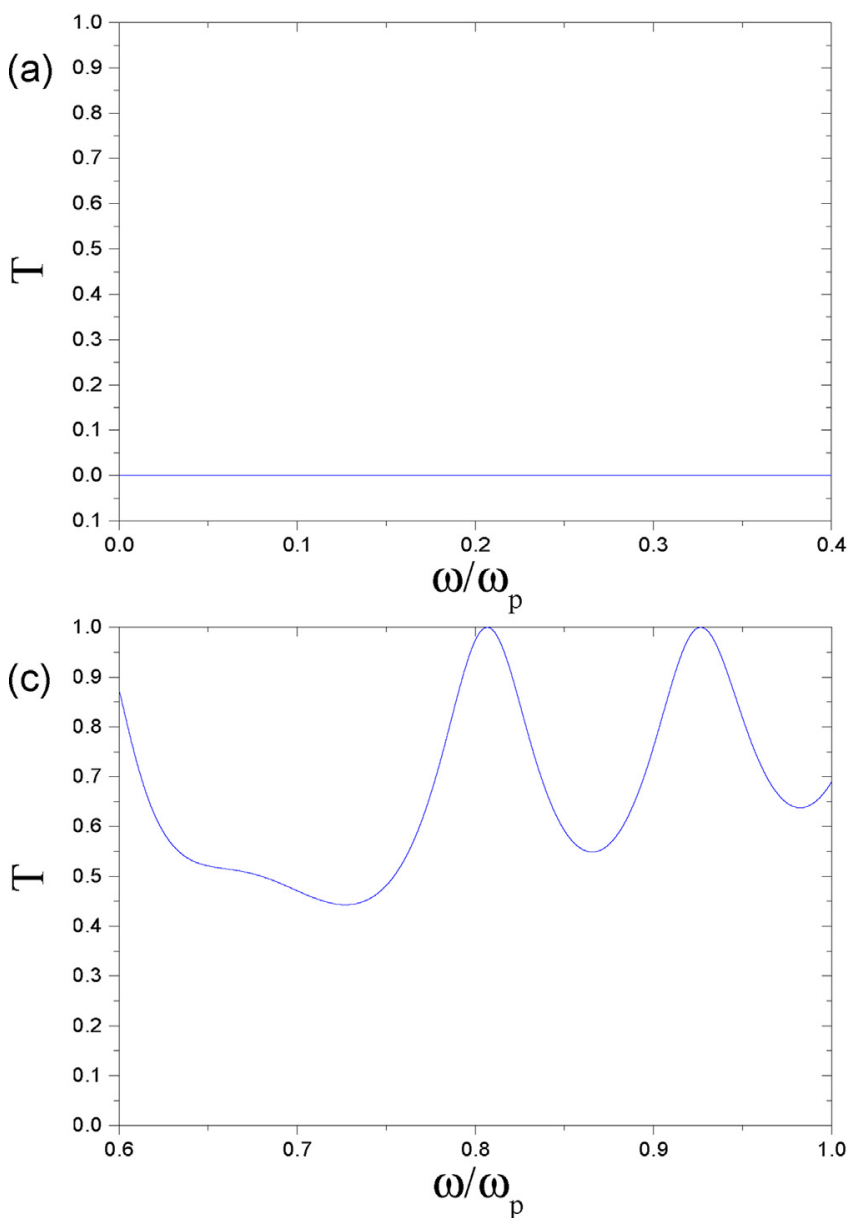

$$
\begin{aligned}
& \vec{E}_{j}^{(N)}=\left(0, E_{y j}^{(N)}, 0\right) \\
& E_{y j}^{(N)}=\left[A_{1 j}^{(N)} \exp \left(-i k_{z j} z\right)+A_{2 j}^{(N)} \exp \left(i k_{z j} z\right)\right] \times \exp \left(i k_{x} x-i \omega t\right)
\end{aligned}
$$

where $A_{1 j}^{(N)}$ and $A_{2 j}^{(N)}(j=A$ or $B ; N=0,1,2, \cdots)$ are the amplitudes.

Application of Maxwell's electromagnetic boundary conditions at the interface $C / A$, yields

$\left[\begin{array}{l}A_{1 C}^{(0)} \\ A_{2 C}^{(0)}\end{array}\right]=M_{C A}\left[\begin{array}{l}A_{1 A}^{(1)} \\ A_{2 A}^{(1)}\end{array}\right]$.

At the interface $A / B$, we find

$\left[\begin{array}{l}A_{1 A}^{(1)} \\ A_{2 A}^{(1)}\end{array}\right]=M_{A} M_{A B}\left[\begin{array}{l}A_{1 B}^{(3)} \\ A_{2 B}^{(3)}\end{array}\right]$.

Successive application of the matrices $\mathrm{M}$ along the finite structure gives

$\left[\begin{array}{c}A_{1 C}^{(0)} \\ A_{2 C}^{(0)}\end{array}\right]=T_{C A B A B \ldots B C}\left[\begin{array}{l}A_{1 C}^{(N)} \\ A_{2 C}^{(N)}\end{array}\right]$,

where
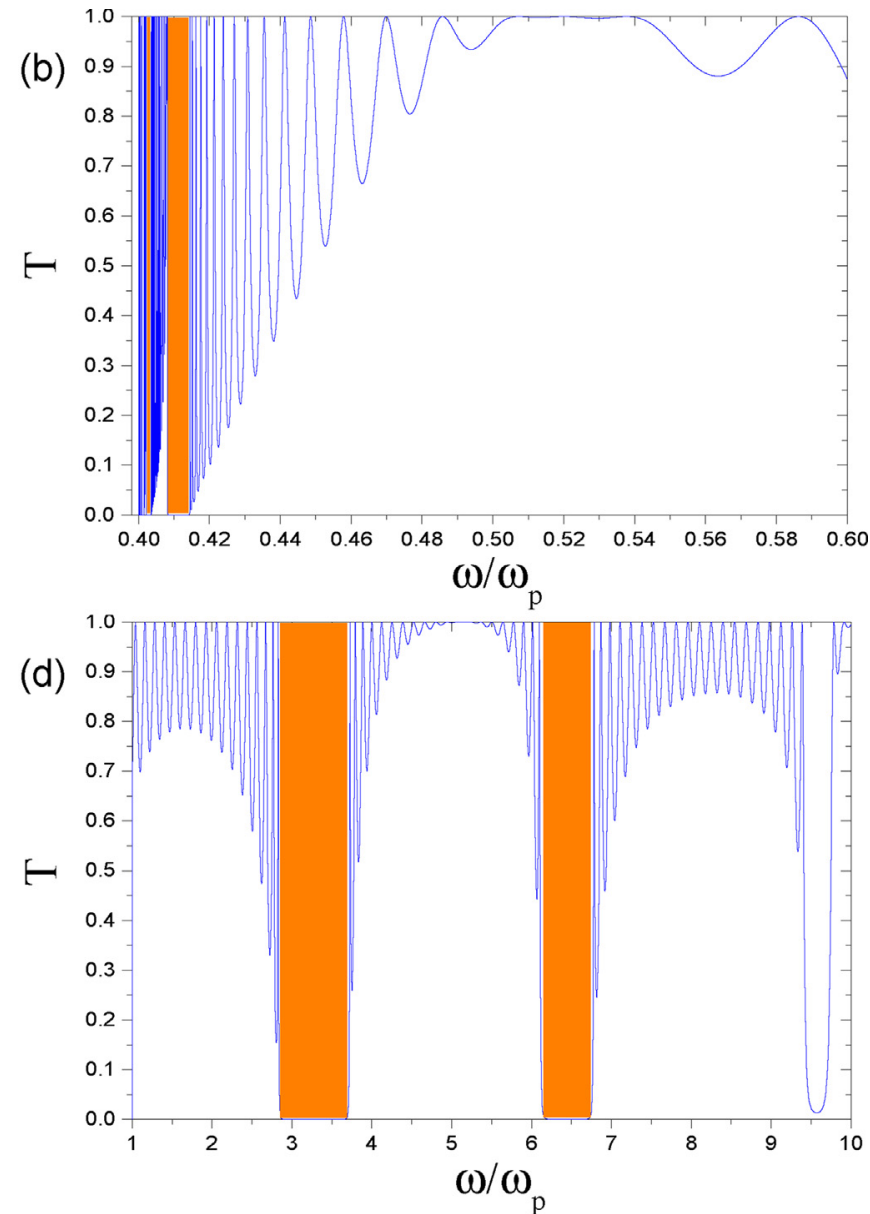

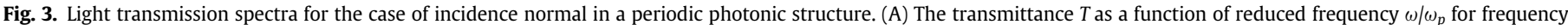

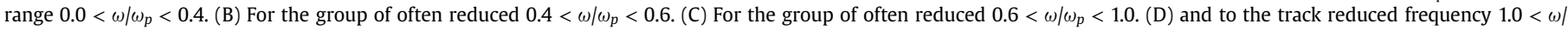
$\omega_{p}<10.0$. 
$T_{C A B A B \cdots B C}=M_{C A} M_{A} M_{A B} M_{B} \ldots M_{B} M_{B C}$.

We now intend to investigate the optical transfer matrices $T$, given by (11) for the periodic case, for structures that exhibit deterministic disorders, i.e. Octonacci structures. Therefore, from Equation (11) we can observe that the transfer matrix $T_{N}$ is formed by a product of matrices $M_{\alpha \beta}$ and $M_{\gamma}$. The ordering of these matrices in the product depends upon the type of quasiperiodic array and the generation number $N$ of the quasiperiodic sequence which is the same index used in the amplitudes of the electromagnetic field. The transfer matrices of all quasiperiodic systems considered here can be straightforwardly determined by induction method [38]. Therefore, by following the Octonacci sequence, it is easy to show that these matrices are given by

$T_{N}=\left(T_{N-1}\right)^{2} T_{N-2}$ for $N$ even,

$T_{N}=\left(T_{N-1} M_{B A}\right)^{2} T_{N-2} \quad$ for $N$ odd,

whose initial conditions are $T_{1}=M_{A}$, and $T_{2}=M_{A} M_{A B} M_{B}$.

\section{Numerical results}

We have chosen medium $A$ as silicon dioxide $\left(\mathrm{SiO}_{2}\right)$, whose refraction index is $n_{A}=1.45$, while medium $B$ is a metamaterial, considering $n_{B}$ as frequency-dependent. Also, we assume the individual layers as quarter-wave layers, for which the quasiperiodicity is expected to be more effective [46], with the central wavelength $\lambda_{0}=700 \mathrm{~nm}$. These conditions yield the physical thickness $d_{J}=\left(175 / n_{J}\right) \mathrm{nm}, J=A$ or $B$, such that $n_{A} d_{A}=n_{B} d_{B}$, which gives the very reversed phase shift in the two materials.

We consider the case where all realized artificial negative refractive index metamaterials have an electric permittivity $\varepsilon$ and a magnetic permeability $\mu$ that are frequency dispersive. Neglecting any damping term, the corresponding dielectric permittivity $\varepsilon(\omega)$ and magnetic permeability $\mu(\omega)$ are respectively given by Ref. [47].

$\varepsilon(\omega)=1-\omega_{p}^{2} / \omega^{2}$,

$\mu(\omega)=1-F \omega^{2} /\left(\omega^{2}-\omega_{0}^{2}\right)$,

where the plasma frequency $\omega_{p}$, the resonance frequency $\omega_{0}$, and the fraction $F$ are given by $\omega_{0} / 2 \pi=4 \mathrm{GHz}, \omega_{p} / 2 \pi=10 \mathrm{GHz}$, and $F=0.56$ (see Ref. [2]).

Fig. 2 shows the plot of the permittivity, permeability, and real and imaginary part of the refractive index versus the reduced frequency $\Omega=\omega / \omega_{p}$. In the present work, investigations have been carried out in yellow and green regions. As frequency increases, four important regions of investigation have been observed. In the white region, $\varepsilon_{B}<0$ and $\mu_{B}>0$. In the yellow region, $\varepsilon_{B}, \mu_{B}$ and refractive index $n_{B}$ are negative. In the green region, $\varepsilon_{B}, \mu_{B}$ and refractive index $n_{B}$ are positive.

Fig. 3 shows the transmission spectrum of electromagnetic radiation, as a function of reduced frequency $\omega / \omega_{p}$, for $s$ polarization (TE wave) and a normally incident wave, for the periodic case at intervals of 20 unit cell repeats in four main regions frequencies shown in Fig. 2 in the range of omega frequency $0.0<\omega / \omega_{p}<10.0$. Polarization $p$ (TM wave) is not taken into account here, because in both polarizations $S$ and $p$ is obtained the same results standards. In Fig. 3 to where refractive index $n_{B}$ is positive and imaginary in the frequency range $0.0<\omega / \omega_{p}<0.4$, shows a complete reflection (Bragg reflector). In Fig. 3 b where $n_{B}<0$ in $0.4<\omega / \omega_{p}<0.6$, shows complete band gaps in the tracks frequency $0.4003 \leq \omega /$

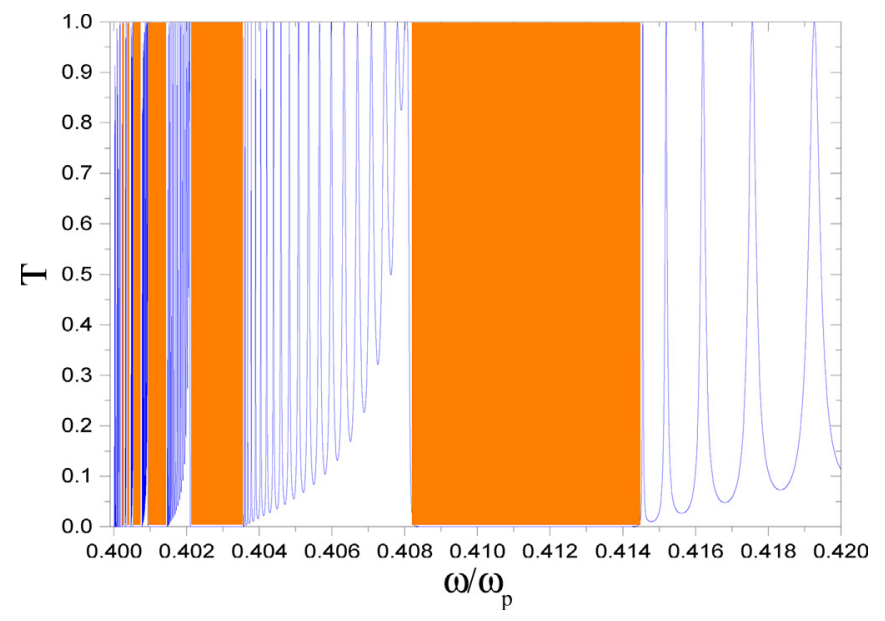

Fig. 4. Light transmission spectra of Fig. $3 \mathrm{~b}$ in the frequency range $0.4 \leq \omega / \omega_{p}=0.42$.

$\omega_{p}=0.4004,0.4005 \leq \omega / \omega_{p}=0.4007,0.4009 \leq \omega / \omega_{p}=0.4010$, $0.402 \leq \omega / \omega_{p}=0.403$ and $0.408 \leq \omega / \omega_{p}=0.414$, which can be observed with more details in Fig. 4 also We observe the Bragg peaks. Fig. 3 c, where $n_{B}$ is positive and imaginary in $0.6<\omega /$ $\omega_{p}<1.0$, It shows only some Bragg peaks. In the end, Fig. $3 \mathrm{~d}$ where $n_{B}>0$ in $1.0<\omega / \omega_{p}<10.0$ shows the complete band gaps in frequency bands $2.84 \leq \omega / \omega_{p}=3.71$ and $6.13 \leq \omega / \omega_{p}=6.76$, and some
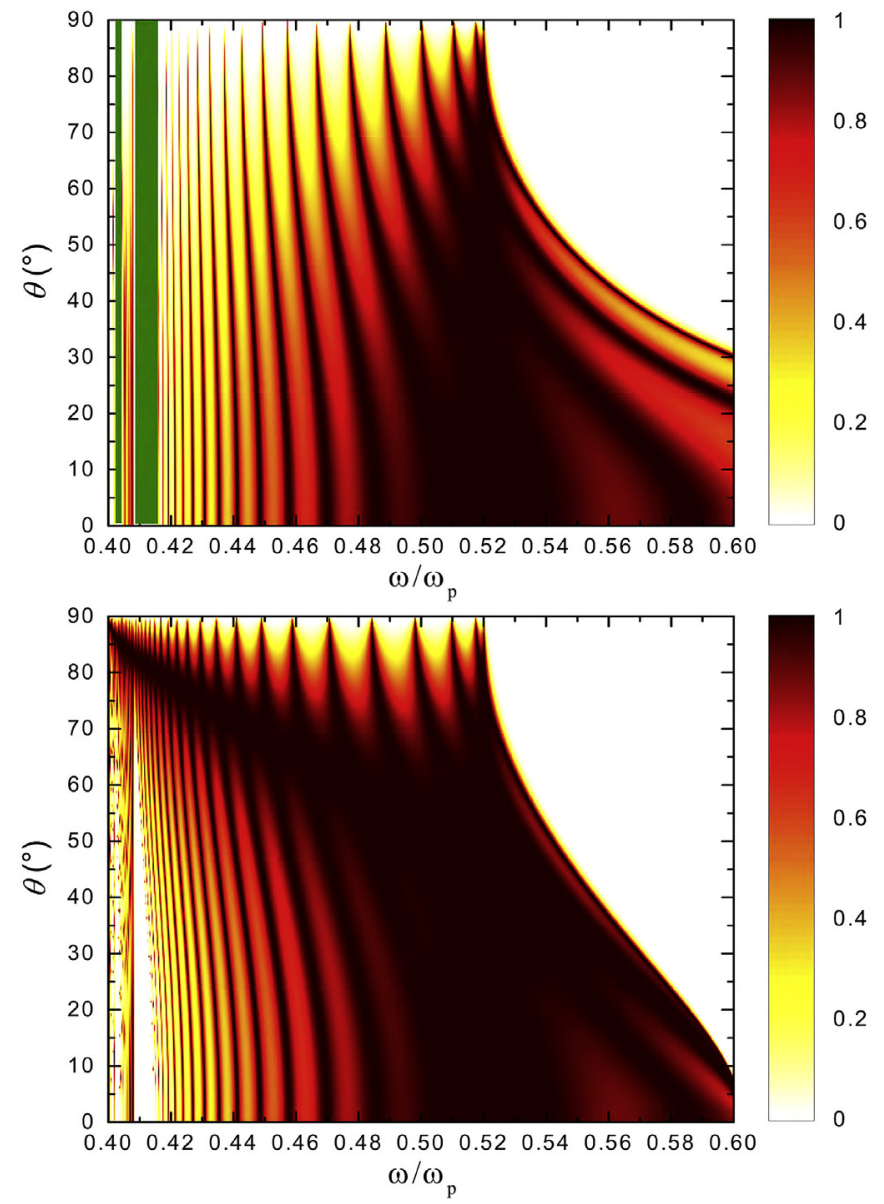

Fig. 5. Transmittance $T$ in color map (see color scale at left), as a function of the reduced frequency $\omega / \omega_{p}$ and incidence angle $\theta$ for the periodic sequence for light polarizations. (a) $s$ polarization (TE waves) (b) $p$ polarization (TM waves). 
Bragg peaks They are also observed.

The transmission spectrum for the periodic sequence analyzed depending on both the reduced frequency $\omega / \omega_{p}$ and incidence angle $\theta=\theta_{C}$ is shown in Fig. 5 for TE and TM polarizations. In Fig. 5a (TE waves) we observe that with the increasing of the incidence angle, the complete band gaps keep the same width in the frequency region $\omega / \omega_{p}$ where $n_{B}$ has considerable negative values. For all angles $\theta>30^{\circ}$ it arises a Bragg pseudo reflector (Bragg mirror), where the refraction index $n_{B}$ tends to zero for $\omega$ close to $6 \mathrm{GHz}$ (see Fig. 2). On Fig. 5b (TM waves) the complete band gaps are no longer seen, and we observe only the Bragg pseudo reflector near the frequencies $\omega / \omega_{p}=0.4$ and $\omega / \omega_{p}=0.6$ when $\theta>9^{\circ}$.

We now proceed to analyze the optical transmission spectrum, for the Octonacci quasiperiodic sequence (OQS), as a function of the reduced frequency $\omega / \omega_{p}$, considering normal incidence for the three regions of Fig. 2, separately. For the frequency range $0.0<\omega /$ $\omega_{p}<0.4$, in the periodic sequence, where $n_{B}>0$, a complete reflection occurs. The same is observed for the Octonacci sequence, irrespective of the generation considered. In Fig. 6 we show the transmission spectra for the fifth, sixth, seventh and eighth Octonacci generations (with $P_{5}=17, P_{6}=41, P_{7}=99$ and $P_{8}=239$ layers, respectively), for $s$ polarization (TE waves) and normal incidence where $n_{B}<0$, which depends on frequency, on the frequency range $0.4<\omega / \omega_{p}<0.6$. One can see that the spectra are not self-similar. Instead of a symmetric distribution, it has three Bragg gaps or complete band gaps, which are characteristic of photonic crystals and conventional Bragg reflectors [39], when compared to quasiperiodic structures found by Medeiros and colleagues [40]. Notice that the complete band gaps are centered around $4.09 \mathrm{GHz}$, $4.24 \mathrm{GHz}$ and $4.44 \mathrm{GHz}$, as shown in the orange (color online) emphasized areas. Also, the light transmission spectra present several Bragg peaks, whose number increases with the generation number $N$. These Bragg peaks where $T=1$ denote a completely transparent system (of course we are considering a lossless case!). On these special peaks we have the famous superlenses, a phenomenon studied by Pendry and Ziolkowski et al. [4,9]. Notice that this intriguing phenomenon would occur in the interval where the refraction index is negative.

In Fig. 7 we show the transmission coefficients for the OQS as a function of the reduced frequency $\omega / \omega_{p}$ and of the incidence angle $\theta=\theta_{C}$ ( $C$ is the vacuum where the light beam comes from), in four situations: fifth (Fig. 8a), sixth (Fig. 8b), seventh (Fig. 8c), and eighth generation (Fig. 8d), respectively, in the frequency range $0.4<\omega /$ $\omega_{p}<0.6$. One can see narrow regions with a well defined photonic band gap. These band gaps are independent of the incidence angle, and are located around the same region of the reduced frequency $\left(0.408 \leq \omega / \omega_{p}=0.410,0.421 \leq \omega / \omega_{p}=0.424,0.438 \leq \omega / \omega_{p}=0.442\right)$ and they have approximately the same width. Again, we can assume the arising of these band gaps are due to the long range order of the arrangement of the layers in the OQS. For all angles $\theta>20^{\circ}$ a Bragg pseudo reflector arises. In Fig. 8 we present the same analysis as Fig. 7, but now for the TM polarization. In this case there are no band gaps. Also, for all angles $\theta>9^{\circ}$ a Bragg pseudo reflector is observed.
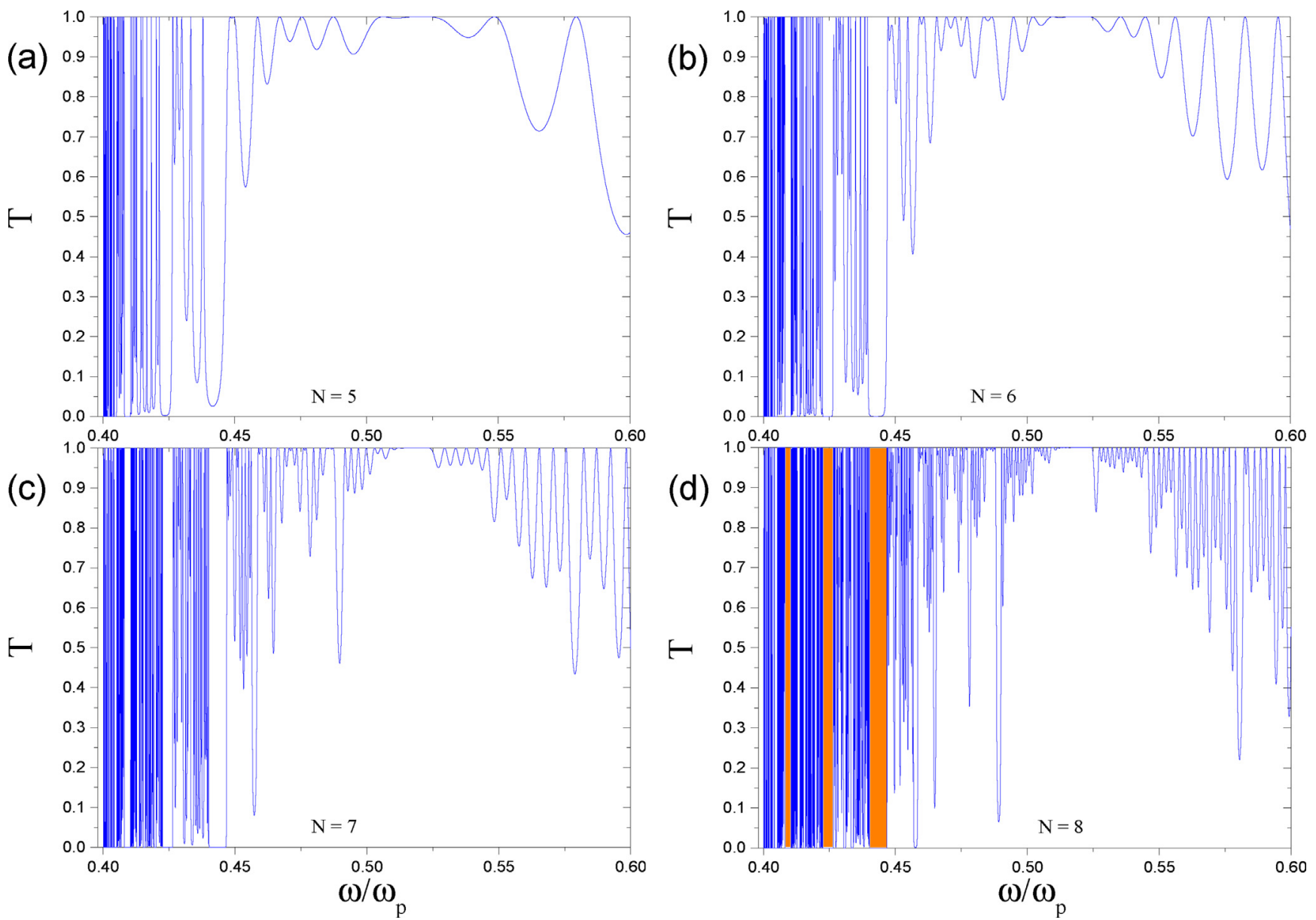

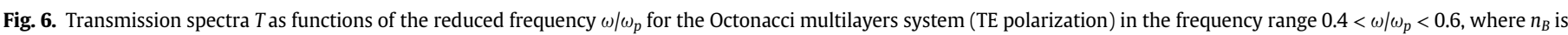
negative. 

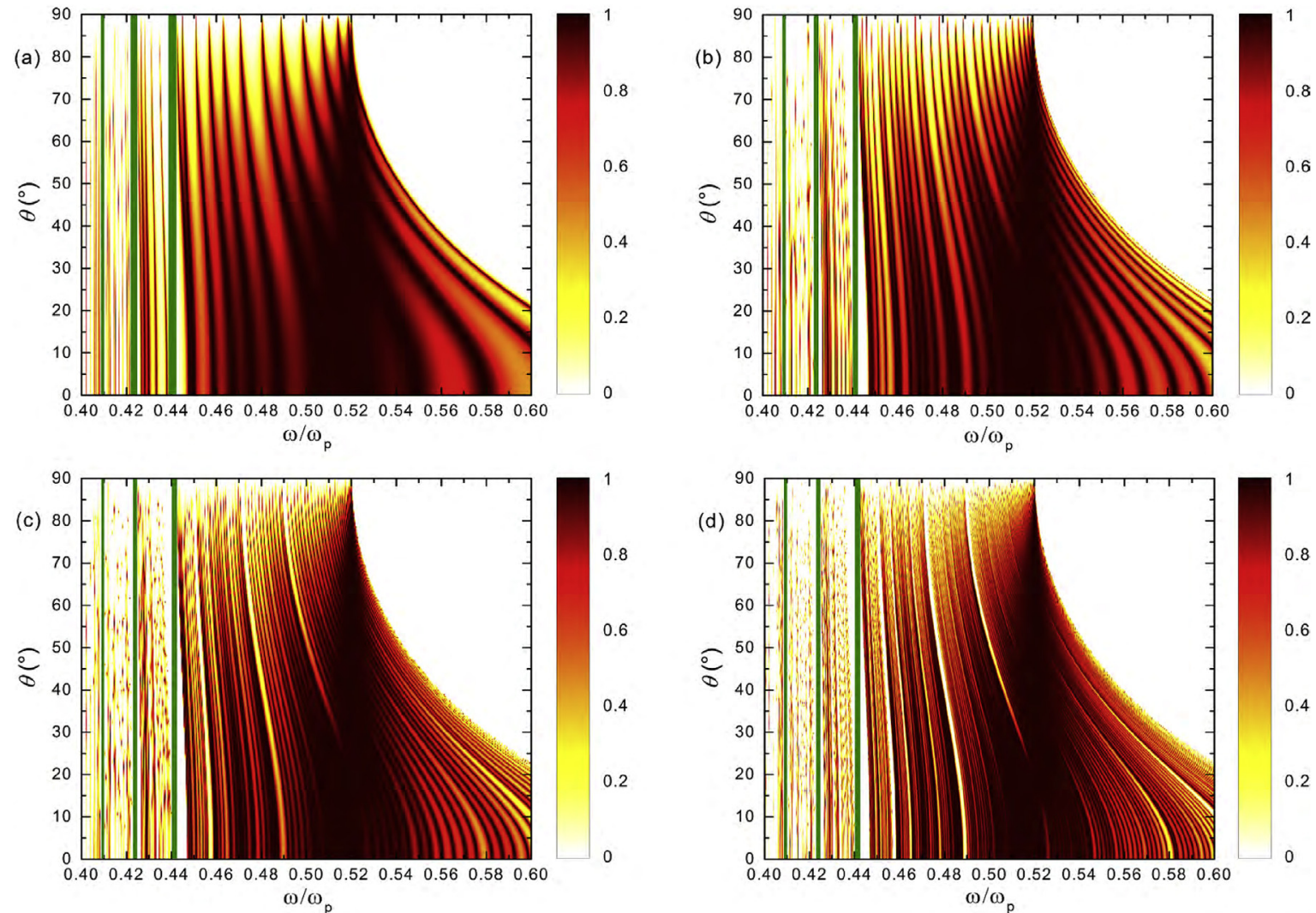

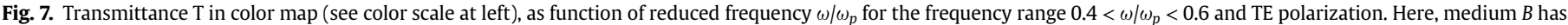

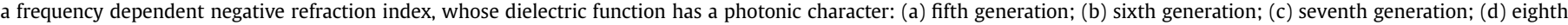
generation of the Octonacci quasiperiodic sequence.
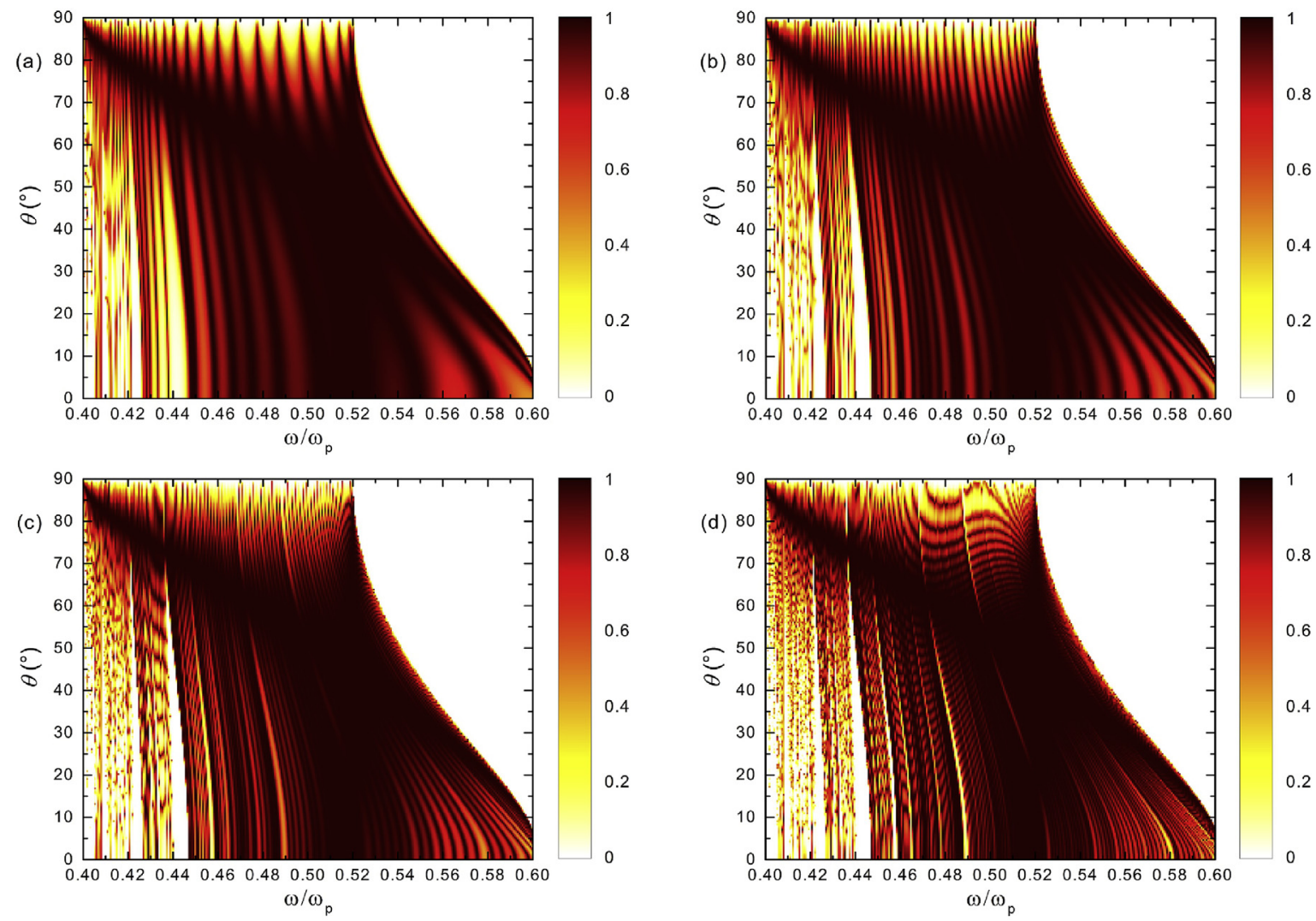

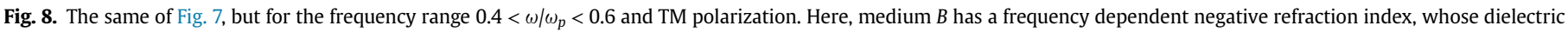
function has a photonic character: (a) fifth generation; (b) sixth generation; (c) seventh generation; (d) eighth generation of the Octonacci quasiperiodic sequence. 

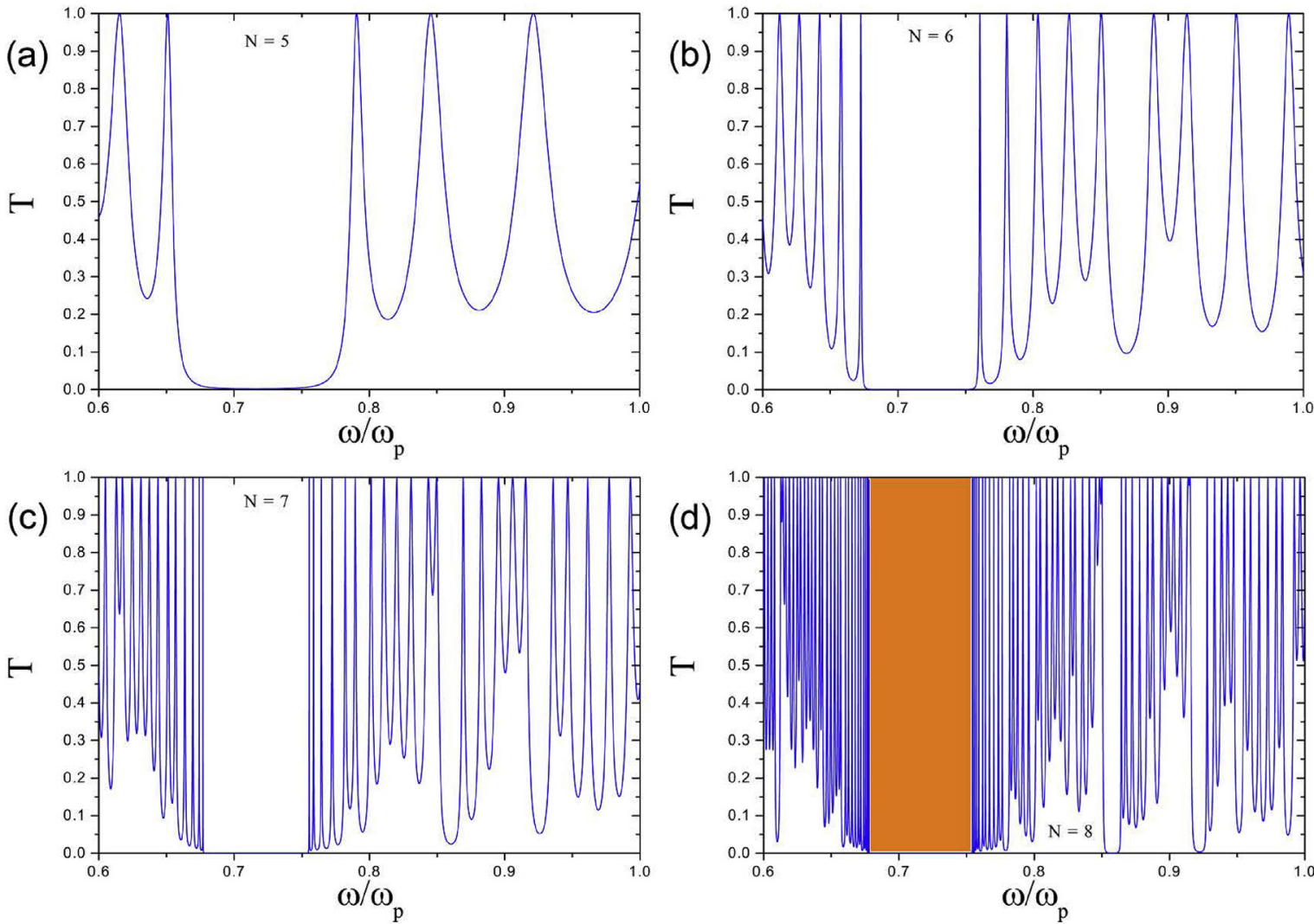

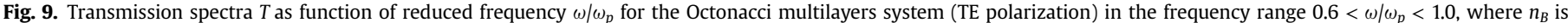
positive (complex).
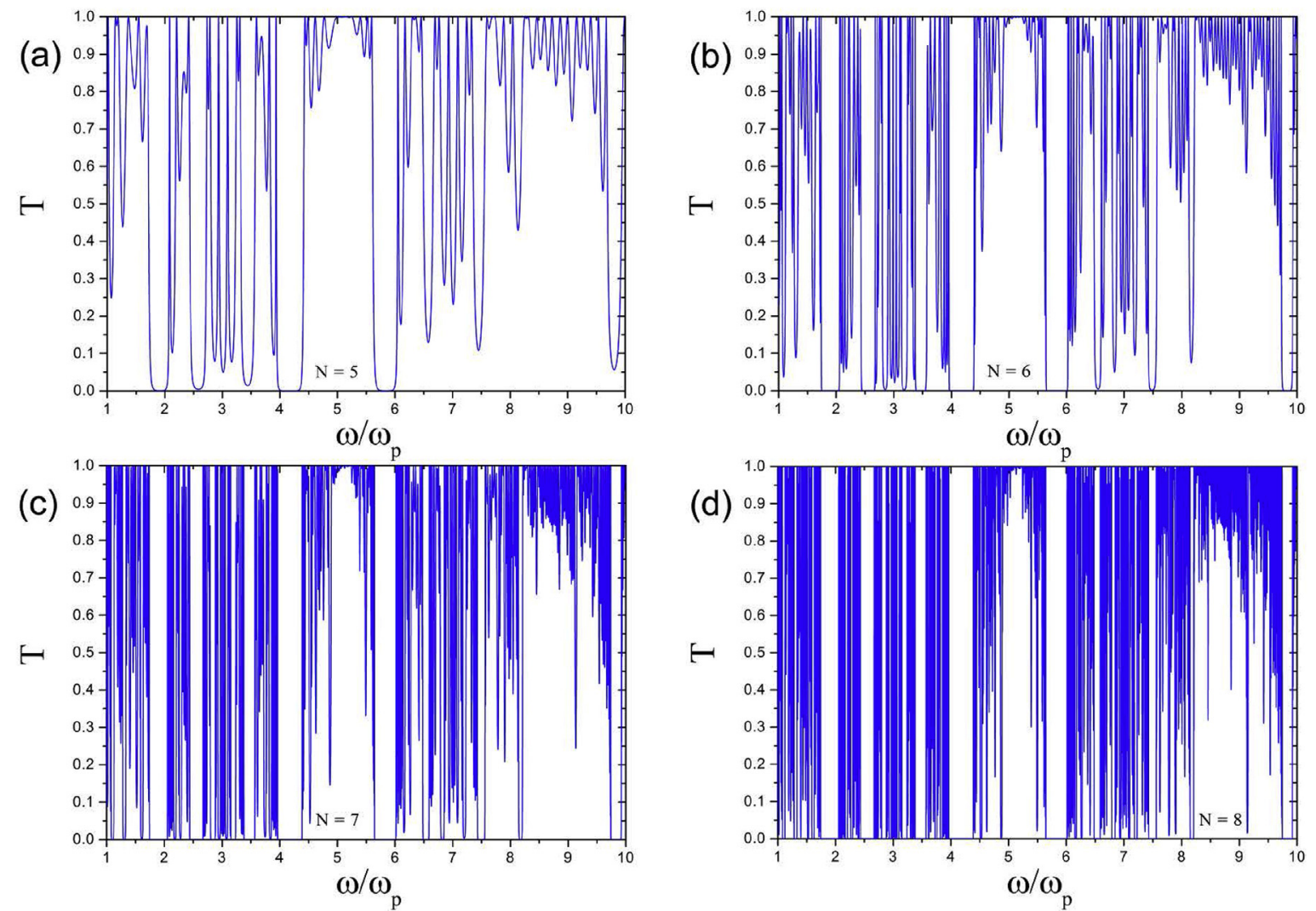

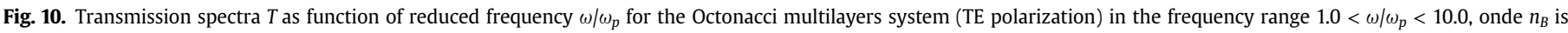
positive (real). 
We have so far analyzed the first two regions of Fig. 2, from a total of four. Now the make an analysis in the third region, where there must be absorption. However, in the present work, in the layer $B$ (metamaterial), where the refraction index depends on the frequency, is a dispersive medium, so it causes some losses. Because of this, the absorption that could be observed is minimal. In the region where $\varepsilon<0, \mu>0$ and $n_{B}>0$ (imaginary), in the frequency range $0.6<\omega / \omega_{p}<1.0$, the light transmission spectrum with normal incidence for the fifth, sixth, seventh and eighth generations of the OQS is shown in Fig. 9a, b, 9c) and 9d, respectively. As one can see, the spectra features a complete transmission, or complete reflection (complete band gap in the frequency range $0.678 \leq \omega / \omega_{p}=0.754$ ) for infinite systems. These features also are noticeable for others frequency range, as $0.1<\omega / \omega_{p}<10.0$, of the OQS (see Fig. 10).

\section{Conclusions}

In summary, we have shown the transmission spectra of light waves propagating through periodic and quasiperiodic Octonacci multilayers. We analysed the four frequency regions, namely, $n_{B}>0$ (imaginary), $n_{B}<0$ (real), $n_{B}>0$ (imaginary) e $n_{B}>0$ (real), respectively, as shown in Fig. 2. Specifically, one of the superlattice components has a negative refraction index, so it is a dispersive medium. In this case, we observe an abrupt behavior of the negative refraction index (NRI) near the frequency $4 \mathrm{GHz}$, and a smooth profile up to the $6 \mathrm{GHz}$ frequency. In the periodic case, the transmission spectra, for normal incidence, presents complete band gaps for some frequency regions and also features several Bragg peaks. When the incidence angle is oblique, for a varying $\theta_{c}$, the band gaps became independent of $\theta_{C}$, for TE polarization, a behavior not observed in the TM polarization. For the quasiperiodic structure, we consider a more realistic situation where the refraction index is frequency-dependent (layer $B$ ). The spectra are also analysed through the four frequency regions, in both normal and oblique incidence. In the frequency region $0.0<\omega / \omega_{p}<0.4$ the spectra presents a complete reflection for all generations of the OQS. Next, in the frequency range $0.4<\omega / \omega_{p}<0.6$, where the refraction index is negative, which means the $B$ layer is a metamaterial, the spectra for normal incidence give rise to a rich profile of transmission of Bragg peaks, without self-similarity or mirror symmetry, but perfect transmission peaks $(T=1)$. When $\theta_{C}$ is allowed to vary, we observe that the band gaps are independent from incidence angle, for TE polarization. For TM polarization the complete band gaps are no longer found. For $0.6<\omega / \omega_{p}<1.0$, where the absorption is small, we have, for normal incidence, a transmission spectre where one can see complete band gaps around $7.16 \mathrm{GHz}\left(0.678 \leq \omega / \omega_{p}=0.754\right)$ for such infinite systems, becoming much more visible for higher generations. On the other hand, for oblique incidence, a complete reflection is more noticeable for TE polarization. However, for TM polarization we found that the spectra is totally reflected (complete reflection), irrespective of the incidence angle. Finally, for the frequency range $1.0<\omega / \omega_{p}<10.0$, the spectra exhibit several Bragg peaks and regions with complete band gaps. These intriguing properties could be used to make resonant optical cavities where a system of Octonacci multilayers (e.g. S4) featuring complete transmission, is built between two optical mirrors. Also we call attention to the possibility of design the so called superlenses studied theoretically by Pendry et al. [4,9], for very particular frequencies. We hope that our work could inspire experimentalists to probe the results presented here.

\section{Acknowledgments}

The authors would like to thank CAPES and CNPq (Brazilian Science Funding Agencies) for the financial support.

\section{References}

[1] V.G. Veselago, The electrodynamics of substances with simultaneously negative values of $\varepsilon$ and $\mu$, Sov. Phys. Usp. 10 (1968) 509-514.

[2] D.R. Smith, W.J. Padilla, D.C. Vier, S.C. Nemat-Nasser, S. Schultz, Composite medium with simultaneously negative permeability and permittivity, Phys, Rev. Lett. 84 (2000) 4184-4187.

[3] R.A. Shelby, D.R. Smith, S. Schultz, Experimental verification of a negative index of refraction, Science 292 (2001) 77-79.

[4] J.B. Pendry, Negative refraction makes a perfect lens, Phys. Rev. Lett. 85 (2000) 3966-3969.

[5] C. Caloz, C.-C. Chang, T. Itoh, Full-wave verification of the fundamental properties of left-handed materials in waveguide configurations, J. Appl. Phys. 90 (2001) 5483-5486.

[6] A.K. Iyer, G.V. Eleftheriades, Negative refractive index metamaterials supporting 2-D waves, in: IEEE MTT International Microwave Symposium (IMS) Digest, 2002. Seattle, WA, 1067-1070.

[7] C. Caloz, H. Okabe, T. Iwai, T. Itoh, Transmission line approach of left-handed materials, in: IEEE AP-S International Symposium and USNC/URSI National Radio Science Meeting, 2002. San Antonio, TX.

[8] I.V. Lindell, S.A. Tretyakov, K.I. Nikoskinen, S. Ilvonen, BW media-Media with negative parameters, capable of supporting backward waves, Microw. Opt. Tech. Lett. 31 (2001) 129-133.

[9] R.W. Ziolkowski, E. Heyman, Wave propagation in media having negative permittivity and permeability, Phys. Rev. E 64 (056625) (2001).

[10] D. Shechtman, I. Blech, D. Gratias, J.W. Cahn, Metallic phase with long-range orientational order and No translational symmetry, Phys. Rev. Lett. 53 (1984) 1951-1953.

[11] N. Wang, H. Chen, K.H. Kuo, Two-dimensional quasicrystal with eightfold rotational symmetry, Phys. Rev. Lett. 59 (1987) 1010-1013.

[12] T. Ishimasa, H.U. Nissen, Y. Fukano, New ordered state between crystalline and amorphous in Ni-Cr particles, Phys. Rev. Lett. 55 (1985) 511-513.

[13] C. Sire, R. Mosseri, Spectrum of $1 \mathrm{D}$ quasicrystals near the periodic chain, J. Phys. Fr. 50 (1989) 3447-3461.

[14] M. Kohmoto, B. Sutherland, C. Tang, Critical wave functions and a Cantor-set spectrum of a one-dimensional quasicrystal model, Phys. Rev. B 35 (1987) 1020-1033.

[15] G. Gumbs, M.K. Ali, Electronic properties of the tight-binding Fibonacci hamiltonian, J. Phys. A 22 (1989) 951-970.

[16] A. Sütő, Singular continuous spectrum on a cantor set of zero Lebesgue measure for the Fibonacci Hamiltonian, J. Stat. Phys. 56 (1989) 525-531.

[17] M. Baake, U. Grimm, R.J. Baxter, A critical Ising model on the Labyrinth, Int. J. Mod. Phys. B 8 (1994) 3579-3600.

[18] E.Y. Vedmedenko, H.P. Oepen, J. Kirschner, Decagonal quasiferromagnetic microstructure on the penrose tiling, Phys. Rev. Lett. 90 (2003) 1372031

[19] B. Chen, C. Gong, The properties of one-dimensional quasiperiodic Lattice's phonon spectrum, Z. Phys. B Condens. Mater. 69 (1987) 103-109.

[20] F. Nori, J.P. Rodriguez, Acoustic and electronic properties of one-dimensional quasicrystals, Phys. Rev. B 34 (1986) 2207-2211.

[21] R. Ilan, E. Liberty, S.E.-D. Mandel, R. Lifshitz, Electrons and phonons on the square Fibonacci tiling, Ferroelectrics 305 (2004) 15-19.

[22] R. McGrath, U. Grimm, R.D. Diehl, The forbidden beauty of quasicrystals, Phys. World 17 (2004) 23-27.

[23] W. Steurer, D. Sutter Widmer, Photonic and phononic quasicrystals, J. Phys. D. Appl. Phys. 40 (2007) R229-R247.

[24] A. Bahabad, R. Lifshitz, N. Voloch, A. Arie, Nonlinear photonic quasicrystals for novel optical devices, Philos. Mag. 88 (2008) 2285-2293.

[25] E. Maciá, Exploiting quasiperiodic order in the design of optical devices, Phys. Rev. B 63 (2001) 205421.

[26] J. Hendrickson, B.C. Richards, J. Sweet, G. Khitrova, A.N. Poddubny, E.L. Ivchenko, M. Wegener, H.M. Gibbs, Excitonic polaritons in Fibonacci quasicrystals, Opt. Express 16 (2008) 15382-15387.

[27] M. Kohmoto, B. Sutherland, K. Iguchi, Localization of optics: quasiperiodic media, Phys. Rev. Lett. 58 (1987) 2436-2438.

[28] K. Esaki, M. Sato, M. Kohmoto, Wave propagation through Cantor-set media: chaos, scaling, and fractal structures, Phys. Rev. E 79 (056226) (2009).

[29] H. Yin, X. Yang, Q. Guo, S. Lan, Optical transmission through generalized SML superlattices, J. Phys. Cond. Mater. 19 (2007) 356221.

[30] W. Gellermann, M. Kohmoto, B. Sutherland, P.C. Taylor, Localization of light waves in Fibonacci dielectric multilayers, Phys. Rev. Lett. 72 (1994) 633-636.

[31] R. Nava, J.T. Martinez, J.A. del Ro, G.G. Naumis, Perfect light transmission in Fibonacci arrays of dielectric multilayers, J. Phys. Cond. Mater. 21 (2009) 155901.

[32] P.W. Mauriz, E.L. Albuquerque, M.S. Vasconcelos, Optical transmission spectra in symmetrical Fibonacci photonic multilayers, Phys. Lett. A 373 (2009) 496-500.

[33] H. Yin, X. Yang, Q. Guo, S. Lan, Optical transmission through generalized SML superlattices, J. Phys. Condens. Matter 19 (2007) 356221

[34] M.S. Vasconcelos, E.L. Albuquerque, Plasmon polaritons and optical spectra of a superlattice of cantor type, Phys. B 222 (1996) 113-122.

[35] M.S. Vasconcelos, F.F. de Medeiros, E.L. Albuquerque, Transmission spectra in photonic band-gap Fibonacci nanostructures, Surf. Sci. 601 (2007) 4492-4496.

[36] C.A.A. Araújo, P.W. Mauriz, E.L. Albuquerque, M.S. Vasconcelos, Phonon 
polaritons in photonic crystals at terahertz frequency range, J. Opt. Soc. Am. B 26 (2009) 1129-1136.

[37] M.S. Vasconcelos, P.W. Mauriz, F.F. de Medeiros, E.L. Albuquerque, Photonic band gaps in quasiperiodic photonic crystals with negative refractive index, Phys. Rev. B 76 (2007) 165117.

[38] M.S. Vasconcelos, E.L. Albuquerque, A.M. Mariz, Optical localization in quasiperiodic multilayers, J. Phys.:Condens. Mater. 10 (1998) 5839-5849.

[39] P. Yeh, Optical Waves in Layered Media", Wiley Series in Pure and Applied Optics, Wiley, New York, USA, 2005.

[40] F.F. de Medeiros, E.L. Albuquerque, M.S. Vasconcelos, Optical transmission spectra in quasiperiodic multilayered photonic structure, J. Phys. Cond. Mater. 18 (2006) 8737-8747.

[41] J. Li, D. Zhao, Z. Liu, Zero-n photonic band gap in a quasiperiodic stacking of positive and negative refractive index materials, Phys. Lett. A 332 (2004)
461-468.

[42] W. Steurer, S. Deloud, Crystallography of Quasicrystals - Concepts, Methods and Structures, Springer, 2009.

[43] E.R. Brandao, C.H. Costa, M.S. Vasconcelos, D.H.A.L. Anselmo, V.D. Mello Octonacci photonic quasicrystals, Opt. Mater. 46 (2015) 378-383.

[44] M. Wu, H. Hung, C. Wu, S. Chang, Analysis of photonic bandgap structure for a polaritonic photonic crystal in negative-index region, J. Opt. Soc. Am. B 31 (2014) 1730-1734.

[45] M.S. Vasconcelos, E.L. Albuquerque, Transmission fingerprints in quasiperiodic dielectric multilayers, Phys. Rev. B 59 (1999) 11128-11131.

[46] K. Kono, S. Nakada, Y. Narahada, Y. Ootuka, Transmission spectra of third sound in a Fibonacci lattice, J. Phys. Soc. Jpn. 60 (1991) 368-371.

[47] H.V. Shadrivov, A.A. Sukhorukov, Y.S. Kivsha, Beam shaping by a periodic structure with negative refraction, Appl. Phys. Lett. 82 (2003) 3820-3822. 